\title{
ECACEN
}

\section{PERSPECTIVAS PARA LA IMPLEMENTACIÓN DE LA CADENA HORTOFRUTÍCOLA EN EL MUNICIPIO DE PENSILVANIA CALDAS \\ HACIA EL AÑO 2025}

\section{PERSPECTIVES FOR THE IMPLEMENTATION OF THE FRUIT HORTICULTURAL CHAIN IN THE MUNICIPALITY OF PENSILVANIA CALDAS TOWARDS THE YEAR 2025}

\author{
Carlos Alberto Arango Álzate ${ }^{1}$ \\ Martha de Jesús Guerrero Guzmán² \\ Martha Lucía Fuertes Díaz ${ }^{3}$
}

\section{Resumen}

La presente investigación tiene como objetivo determinar las perspectivas y estrategias aplicables, para la implementación de la Cadena Hortofrutícola en el municipio de Pensilvania Caldas con visión de rendimiento al ciento por ciento en el año 2025.

Se llevó a cabo una investigación exploratoria o preliminar en donde se utilizó la observación de campo, para diagnosticar la situación actual, cambios que se han

\footnotetext{
${ }^{1}$ Profesional en ciencias administrativas, Maestrante en Administración de organizaciones UNAD.

2 Docente Universidad Nacional Abierta y a Distancia. Magíster en administración de empresas y liderazgo estratégico.

3 Doctoranda en Administración Gerencial, Magister en Administración con Énfasis en Finanzas Corporativas, Especialista en Finanzas con concentración en Finanzas Avanzadas, Especialista en formación del aprendizaje con base en competencias laborales, Contadora Pública. Docente de la Universidad Nacional Abierta y a Distancia. Investigadora y Directora del Grupo de investigación ilama. ORCID http://orcid.org/0000-0001-8468-1867 Google Scholar: https://scholar.google. com.co/citations?user=IP5s- PcAAAAJ\&hl=es Correo electrónico: martha.fuertes@unad.edu.co y marthalfd@yahoo.com
} 


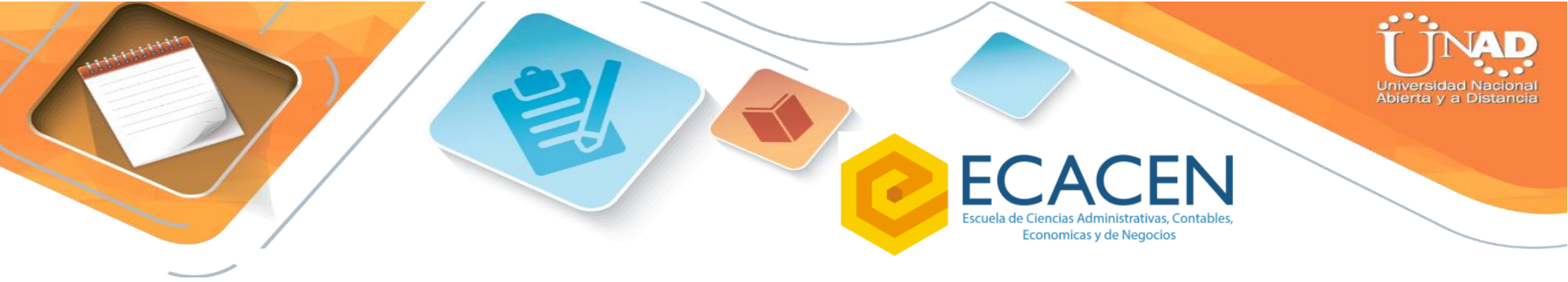

El presente documento en una primera parte contiene los temas que se refieren a la problemática que se presenta, los objetivos del trabajo y la justificación que amerita el estudio. En una segunda parte se encuentra el marco teórico que soporta la investigación. En tercer lugar, se relacionan las estrategias para el desarrollo de la cadena hortofrutícola en Pensilvania Caldas. Como cuarto aspecto se contemplan los sistemas de producción, y finalmente las conclusiones y recomendaciones emergidas del proyecto.

La información disponible será el insumo para concertar la ejecución de una factible implantación de la Cadena Productiva Hortofrutícola en el municipio de Pensilvania, con visión hacia el año 2025.

\section{Agroindustria en Caldas}

Uno de los principales problemas de la agroindustria en Caldas es la disponibilidad de la materia prima (frutas, verduras y otras), hay muchos altibajos en la producción, y todo debido a que no se da de acuerdo a unas necesidades establecidas por la agroindustria y la comercialización, presentándose verdaderas sobreofertas unas veces, y otras, gran escasez; estos altibajos en la producción, las prácticas inadecuadas de cultivo y poscosecha, sin tener en cuenta la aptitud del suelo, impiden que este obtenga mejores ingresos por sus esfuerzos.

El sector hortofrutícola tiene un futuro promisorio en término de los mercados abiertos gracias a los tratados internacionales, y a su participación en las generaciones de riqueza y empleo, dadas sus especiales condiciones para la agregación de valor.

Pero también, es inocultable la crisis que experimenta el sector agropecuario colombiano, que se endurece sobretodo en el productor primario, las regiones buscan alternativas económicas que contribuyan al desarrollo empresarial y a mejorar la 


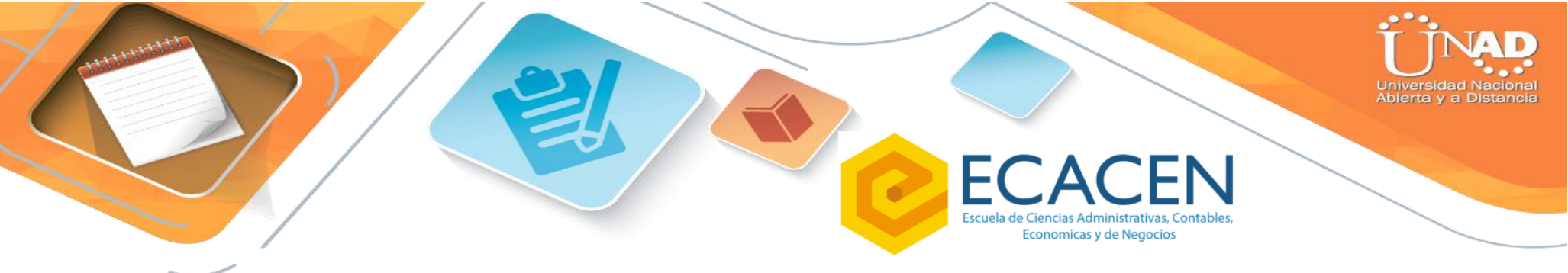

rentabilidad del campo. Entre esas alternativas surge la Cadena Hortofrutícola de Pensilvania como una opción importante dadas sus características y perspectivas.

Con la presente investigación se pretende presentar las perspectivas y estrategias, para la implementación de la Cadena Hortofrutícola en el municipio de Pensilvania con enfoque agregado de competitividad, para desarrollar esta cadena integralmente, asegurando su viabilidad económica, ambienta, social, cultural.

Se le quiere dar vida a una idea factible en donde agentes empresariales e institucionales públicos y privados tendrán que llegar a compromisos, y a definir estrategias que permitan formalizar una cadena productiva que logre alcanzar índices competitivos de calidad, precio y oportunidad para los productos.

El resultado del estudio, fruto de los objetivos, llevará a encontrar alternativas concretas de solución, y que puedan materializarse al interior de los eslabones de la cadena productiva hortofrutícola que se quiere implantar.

La cadena productiva hortofrutícola se proyecta como una estrategia para lograr el desarrollo económico, regional, y rural, de ahí el impulso de los actores institucionales al tema en aras de generar el cambio social que urge.

Se busca producir aquella que nos convierta en verdaderos competidores frente a otras regiones que producen lo mismo, en mejores condiciones.

\section{Marco Teórico}




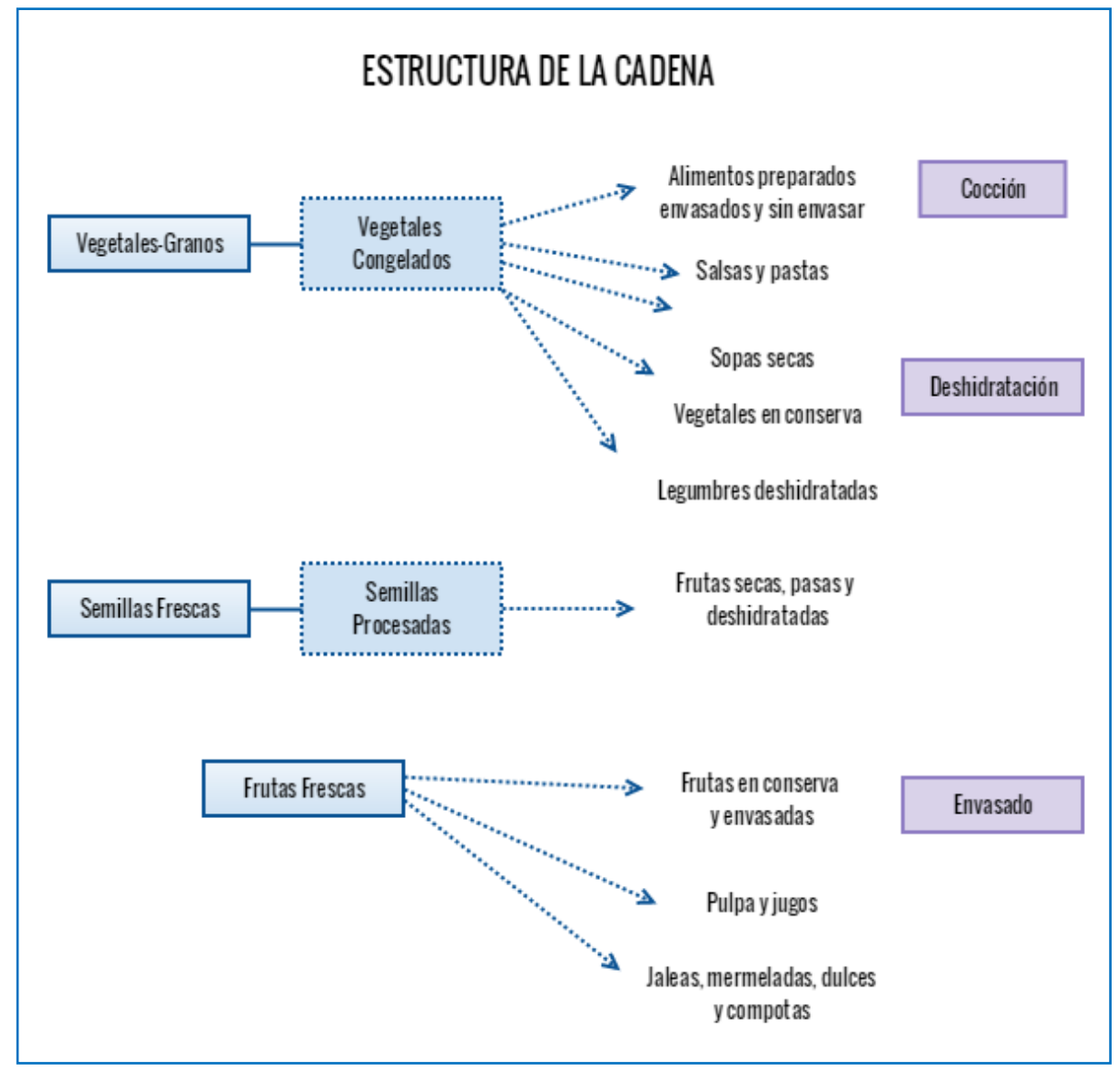

Figura 1: Estructura de la cadena hortofrutícola. Fuente: Elaboración propia. A partir de: (Superintendencia de Industria y Comercio (SIC), Cadena productiva de las hortalizas en Colombia: Diagnóstico de libre competencia, 2011)

\section{Eslabones:}

Frutas frescas, vegetales, granos, vegetales congelados, frutas secas, pasas y deshidratadas, frutas en conservas, pulpa y jugos, jaleas, mermeladas, dulces y compotas; alimentos preparados envasados y sin envasar, salsas y pastas, sopas secas, vegetales en conservas, legumbres deshidratadas, semillas procesadas, arinas. 


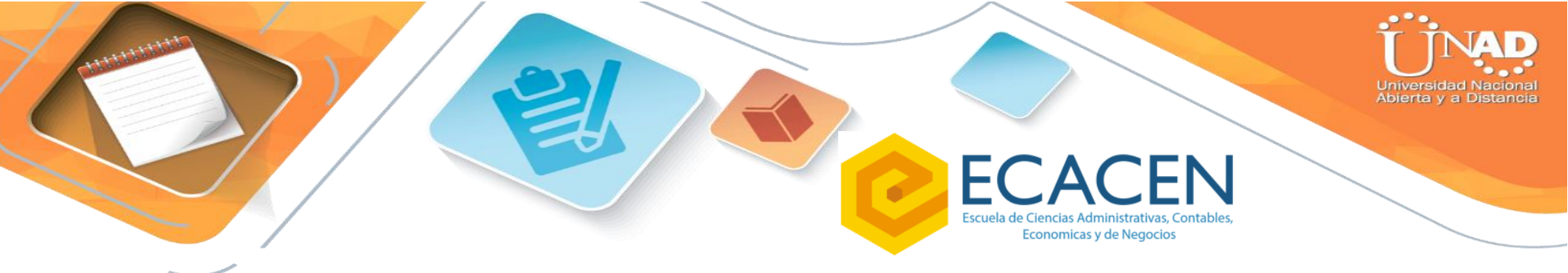

donde deberá estar consolidada por el entorno favorable que se le abre en todas las líneas. Una alimentación correcta implica una cadena de relaciones armónicas y coherentes, para generar cantidad, diversidad y calidad en los alimentos. Estos dan competitividad a la producción agropecuaria, que en muchos lugares carece de valor, porque no hay organización y motivación en la búsqueda de escenarios propicios para defender y estimular la producción agrícola.

\section{Acciones estratégicas por desarrollar a la firma}

Las acciones por desarrollar a la firma de un acuerdo de formalización de la cadena hortofrutícola en Pensilvania, Caldas, se localizarían en niveles de corto, mediano y largo plazo, mediante acuerdo con campesinos que son el factor principal, la alcaldía municipal en a través del su plan de desarrollo, la gobernación de caldas a través de la secretaria de agricultura, el ministerio de agricultura, las asociaciones y ONGS que se tienen en el municipio y que trabajan estos proyectos con la comunidad, se desarrolló de la siguiente forma:

- Concertación de un cronograma entre las partes, con el apoyo del coordinador de la cadena.

- Establecimiento de la mesa de operatividad, con un detallado manual de funciones, deberes, obligaciones y derechos.

- Desarrollo del recurso humano participante.

- Estudio, análisis y concertación de un proceso fuerte y sostenible, cuyo despliegue implicará toma de decisiones y realizaciones en el corto y mediano plazo.

- Definición de claras directrices y metas que permitan la continuidad y crecimiento en condiciones de mercado y competitividad.

- Análisis y estudio de políticas de comercio, inversión, cofinanciación, financiación, desarrollo de infraestructura básica para la producción, ambiental y de recursos humanos. 


\section{Sistemas de Producción}

En el contexto de las cadenas de producción, la metodología de sistemas de producción está aplicándose en el sector agrícola desde hace ya varias décadas en las áreas de investigación, transferencia tecnológica y asistencia técnica. Es fundamental abordar este tema afín a las cadenas de producción, porque los sistemas de producción buscan analizar la problemática y potencialidad de las cadenas de producción, a través de una visión integral de los componentes constituyentes de estas.

\subsection{Conceptualización}

"El sistema es el conjunto de elementos o componentes en interacción dinámica, organizados en función de una finalidad u objetivo común". (Duarte, 1996). Esto corresponde a un análisis integral que deberá hacerse, para comprender más fácilmente los fenómenos biológicos.

"Un sistema de producción agropecuario es un conjunto de actividades que un grupo humano organiza, dirige y realiza de acuerdo con sus objetivos, cultura, recursos, utilizando distintas prácticas tecnológicas, en respuesta al medio físico para obtener diferentes producciones agrícolas". (Duarte, 1996). También se habla de la forma como se combina los factores de producción: tierra, trabajo, y capital, gestión realizada por el hombre para satisfacer sus propósitos económicos.

\subsection{Características de metodología de sistemas de producción}

Estas características de los sistemas productivos son básicas para el análisis de las cadenas de producción. Se tienen la planificación, discusión y análisis del trabajo, las cuales deben ser ejecutadas en grupos.

De igual forma, se integran diferentes tipos de conocimiento, el empírico del productor, el científico y académico del técnico, y finalmente, el conocimiento público de los representantes políticos.

El escenario fundamental son las condiciones de los productores y su entorno, por ejemplo, la toma de información primaria, el montaje de parcelas, en el marco de los agricultores. 
convenio con comité de cafeteros para renovación de café con entrega de 514.000 chapolas. Se está desarrollando el concurso de cafés especiales, sobre el cual la federación de cafeteros, sacará una versión de Juan Valdez en homenaje a los 150 de fundación de Pensilvania-

- Caña, Cacao, Plátano, Aguacate: Son cadenas productivas que se buscan implementar en mayor nivel en el Municipio, con la gobernación se están haciendo alianzas para que el municipio quede incluido en el proyecto que se va a presentar al fondo de regalías.

- Cacao y Plátano: Con INCODER (En Liquidación), se está adelantando un proyecto para suministrar a una asociación de Pueblo Nuevo, material vegetal para establecer estos cultivos, apoyado con certificación del SENA.

- Plátano: Con la Fundación FUNCOLAGRO, se está apoyando un proyecto sobre establecimiento de un vivero de Plátano y establecimiento de cultivos.

- En términos generales se está buscando en el Municipio, implementar el establecimiento de estos cultivos.

- Seguridad Alimentaria: Ante Prosperidad Social se presentó un proyecto en conjunto con Marquetalia y Manzanares para el tema de soberanía alimentaria; además se están adelantando proyectos de Sena Emprende Rural, en diferentes sectores del Municipio, para dar formación en cultivos transitorios, huertas caseras y suministro de semillas y fertilizantes con el fin de implementar proyectos productivos con la comunidad.

- Comercialización de productos: Se están realizando mensualmente mercados campesinos en el Municipio con el fin de facilitar a los campesinos la venta de sus productos, además de otras estrategias de comercialización.

\section{Conclusiones}

La investigación sobre la perspectiva de una cadena productiva en Pensilvania consolidada hacia el año 2025, ha posibilitado tener una visión muy positiva acerca de 


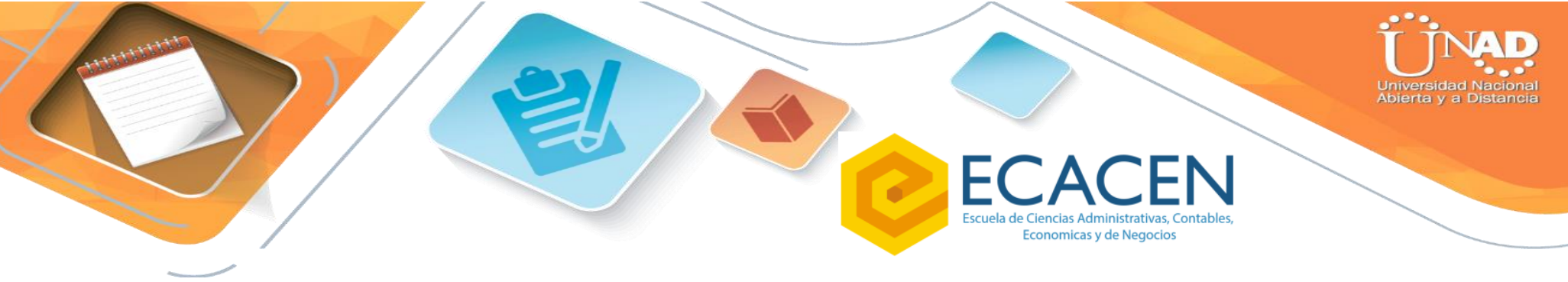

la implementación de la Cadena Hortofrutícola, con énfasis en el cultivo del plátano, en el municipio de Pensilvania, Caldas.

Con base en los resultados de la fase de exploración y la encuesta se pudo constatar que el sector hortofrutícola tiene en la actualidad un gran papel gracias a los mercados abiertos y a los términos de su participación en la generación de riqueza y empleo, dadas sus condiciones especiales para la agregación de valor. Una cadena productiva en esta área en el municipio tiene un futuro promisorio en todos los sectores teniendo en cuenta los potenciales de organización, zonificación, alianzas estratégicas entre productores, comerciantes, agroindustria, organizaciones, que van a asegurar su sostenibilidad y competitividad.

El enfoque de competitividad bajo el cual se aspira a desarrollar esta cadena es de carácter integral, y con capacidad para posicionarse en el mercado local y regional, asegurando su viabilidad económica, ambiental, social y cultural, a través de calidad, precio y oportunidad. En este contexto existe la voluntad expresa de los actores empresariales e institucionales para compartir información, establecer alianzas, cumplir compromisos, y la gestión antes las autoridades municipales, departamentales y nacionales para implementar y posicionar la cadena según los diálogos establecidos con ellos.

Para todos los involucrados, las etapas de la Cadena Hortofrutícola en Pensilvania: recolección, producción, distribución, comercialización, son posibles de llevar a cabo, y tienen una amplia expectativa de hacerse realidad en la práctica.

Un apartado de suma importancia en la presente investigación es el pertinente al plátano, pues la consulta que se realizó con los diferentes sectores señala a este producto como el más importante dentro de la implementación de la cadena hortofrutícola, se analizaron y tuvieron en cuenta varios aspectos:

La cadena agroalimentaria del plátano está conformada por los productores, comercializadores, productores de semilla, proveedores de insumos, exportadores, 


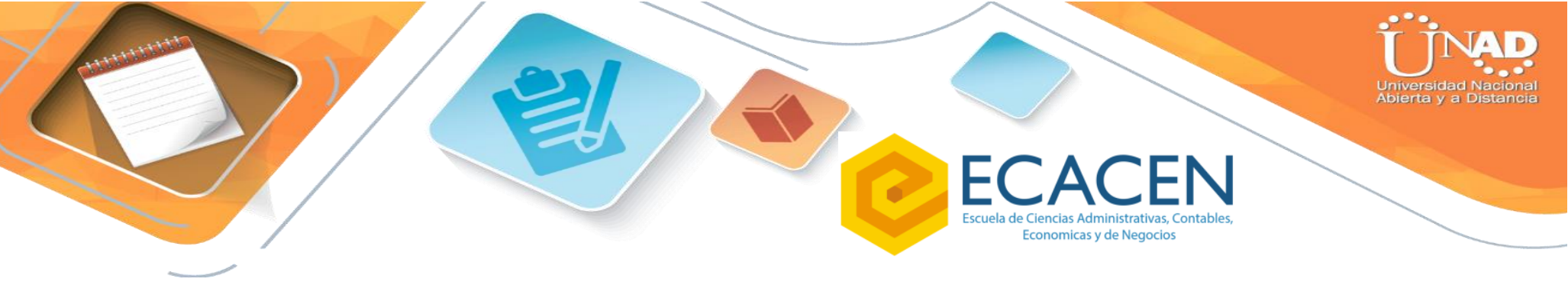

enriquecer experiencias y esfuerzos para implementar la Cadena Hortofrutícola en Pensilvania, hacia el 2025 con énfasis en el plátano.

Todos los actores están dispuestos a trabajar de manera activa y mancomunada para lograr los objetivos propuestos.

Los resultados de la encuesta permiten deducir que: El desarrollo de una cadena hortofrutícola en el municipio de Pensilvania, será una gran alternativa que genera desarrollo y mejorara las calidad de vida de los campesinos del municipio, mediante el estudio se ha podido evidenciar que los campesino cuentan con mano de obra, tierras productivas, climas óptimos, capacidad e interés por asociarse, apoyo de los fundaciones, alcaldía, departamento y nación para lograr que la cadena sea un éxito, además como se planteó en los socios estratégicos que se tienen cuentan con la capacidad de consumir el plátano que sea producido en este región, factor fundamental y que genera confianza y motivación en los productores, ya uno de los principales inconvenientes ha venido siendo el mercado del producto.

Podrá el plátano convertirse en un producto de primer renglón en la economía del municipio lo que generará mejores condiciones económicas y de vida de los campesinos que decidan iniciar este cultivo de una forma tecnificada, asociados para lograr mayores beneficios y aprovechando las oportunidades que están dando el gobierno al campo y a los productores que deseen organizarse; está demostrado que el plátano es un producto rentable y que cuenta con una gran demanda y mercado asegurado.

\section{Referencias}

Departamento Nacional de Planeación. (2004). Cadenas Productivas:Estructura, comercial internacional y protección. Bogotá: DNP.

Duarte, O. (1996). Conceptos básicos sobre la metodología de sistemas de producción. Bogotá: Ediciones ABC. 
Johnston, B., \& Mellor, J. (1974). El papel de la agricultura en el desarrollo económico. México: Edmundo Flórez Editores.

Ley 101 de 1993. (1993). Obtenido de Secretaria de Senado: http://www.secretariasenado.gov.co/senado/basedoc/ley_0101_1993.html

Ley 811 de 2003. (2003). Obtenido de Secretaria de Senado: http://www.secretariasenado.gov.co/senado/basedoc/ley_0811_2003.html

Superintendencia de Industria y Comercio (SIC). (2011). Cadena productiva de las hortalizas en Colombia: Diagnóstico de libre competencia. Obtenido de SIC: http://www.sic.gov.co/drupal/masive/datos/estudios\%20economicos/Document os $\% 20 \% 20$ elaborados $\% 20$ por\%20la\%20Delegatura $\% 20$ de $\% 20$ Protecci $\%$ C3\%B 3n\%20de\%20la\%20Competencia/2011/Hortalizas2012.pdf 